\title{
Bipolar relapse precipitated by dopaminergic agents in Parkinson's Disease: Case report and review of literature
}

\section{Dr Shi Wei Ong, Department of Psychological Medicine, Changi General Hospital, Singapore}

\section{INTRODUCTION}

Dopaminergic agents are used in Parkinson's disease to manage the troubling motor symptoms of dyskinesia, rigidity, and tremors. These include dopamine agonists such as ropinirole and pramipexole, as well as the mono-amine oxidase inhibitor selegiline. Such agents have been implicated in the subsequent development of neuropsychiatric symptoms in Parkinson's disease patients. The following report describes a case of bipolar relapse precipitated by dopaminergic agents in a patient with Parkinson's disease.

\section{CASE REPORT}

Mr P is a 54 years old married male of Chinese ethnicity who was referred to the psychiatry consultation-liaison service of a general hospital in Singapore. He had been admitted to the general medical unit for an acute change in mental status and behavior. Medical workup for organic causes had been negative.

His family reported that for a week, he had been sleeping poorly, and behaving in a disorganized manner in the day, such as constantly rearranging and sorting his belongings, using toilet paper to cover himself, and sitting in the 34 degrees centigrade hot sun for hours. When he did not sleep for three nights in a row and became aggressive towards his daughter, his family decided to bring him to the hospital.

Past medical history was significant for Parkinson's disease diagnosed four months prior to the current presentation. He had been started on ropinirole as well as selegiline. The dosages were increased just a month ago, as his motor symptoms continued to be troubling such that he was forced to stop his food stall business. There was no neuropsychiatric concerns at the time. He has a past psychiatric history of bipolar disorder that had required three inpatient admissions for manic relapses. He would improve quickly with medications, but would be irregularly adherent in between. He stopped taking medications completely for the past ten years, and the illness had been in remission.

When he was seen by the psychiatric team, he was noted to be thought disordered, with speech that frequently derailed needing re-direction and clarification. He expressed paranoid ideas about seeing and hearing little ghosts at home that had followed him to the hospital. They were messing with his belongings and had tried to possess his family. He needed to absorb the power of the sun in order to defend himself against them. When he saw one of them possess his daughter, he attacked it.

Nursing staff reported to have seen him mumbling to himself at times, and behaving in a paranoid fashion, for example, peering under beds and then move his limbs as if performing religious rites.

References:

Berk M. et al. (2007). Dopamine dysregulation syndrome: Implications for a dopamine hypothesis of bipolar disorder. Acta Psychiatr Scand, 116 (suppl 434), 41-49

Engmann B. (2011). Case report: Bipolar affective disorder and Parkinson's disease. Case Reports in Medicine, doi:10.1155/2011/154165

Maier F. et al (2014). Hypomania and mania related to dopamine replacement therapy in Parkinson's disease. Parkinsonism and Related Disorders, 20, 421-7.

Beyer MK et al. (2007). A magnetic resonance imaging study of patients with Parkinson's disease with mild cognitive impairment and dementia with voxel-based morphometry. $J$ Neurol Neurosurg Psy, 78(3), 254-9

Leentjens AFG (2004). Depression in Parkinson's disease: Conceptual issues and clinical challenges. J Geriatr Psychiatry Neurol, 17(3), 120-6.
The psychiatric team's impression was that of psychosis, precipitated by antiparkinsonian medications. He was started on a low dose of quetiapine. Neurology consult was made, with suggestions to stop his current antiparkinsonian medications and switch to levodopa.

Within a week his thought disorder improved. However, his mood was discernibly more expansive, and he started making cheeky, overfamiliar remarks to female staff. He was more energetic, spending a lot of time on the ward's stationary bicycle, writing daily on dining serviettes about various issues, including a plan to restart his food business and detailing all the equipment he will need and how he was going to remodel the stall. He became hyper-religious, chanting, and performing rites. Cognitively he was lucid with no apparent deficit.

His parkinsonian symptoms had been reasonably managed with judicious titration of quetiapine to $100 \mathrm{mg} \mathrm{ON}$, and levodopa. Nonetheless there had been some episodes of postural hypotension. Decision was made not to further increase quetiapine, but to add sodium valproate to stabilize his elevated mood. His manic symptoms settled by two weeks and he was discharged on quetiapine $100 \mathrm{mg}$ ON and sodium valproate $500 \mathrm{mg}$ BD.

Unfortunately, Mr P stopped taking all medications (including his antiparkinsonian medications) two weeks after discharge, and relapsed with more florid manic symptoms (flight of ideas, pressured speech, frank grandiosity) necessitating another round of inpatient admission. Again he improved rapidly with restart of psychotropics.

\section{DISCUSSION}

The relationship between bipolar disorder and Parkinson's disease has been noted in several case reports and one small cohort study (Maier et al, 2014). The pathophysiology underpinnings of this relationship is however, unclear. In Parkinson's disease, there is dopaminergic degeneration, with functional progressive degeneration of the whole brain involving the limbic system and neocortex (Beyer et al, 2007). Clinically this manifests as non-motor symptoms, such as neuropsychiatric disturbances, the most common of which is depression (Leentjens, 2004).

Hence dopaminergic dysfunction has been suggested to be a key contributor (Berk et al, 2007). Evidence for this includes the observation that levodopa or dopaminergic agents given to bipolar patients can produce hypomanic episodes; and that conventional antipsychotics that block dopamine D2 receptors are known to be effective in mania. Conversely, low dopamine levels can lead to depression, with dopaminergic anti-depressants (such as amisulpiride and buproprion) shown to be efficacious.

In our patient $\mathrm{Mr} \mathrm{P}$, it is likely that his pre-existing bipolar disorder relapse was triggered by the dopaminergic overdrive due to the antiparkinsonian agents. There is little evidence available for treatment options of bipolar mania in Parkinson's disease; the usual agents of choice (lithium, atypical anti-psychotics) risk worsening the motor symptoms. Sodium valproate and lamotrigine have been suggested to be reasonable options (Engmann, 2011). At the same time, close collaboration with neurology counterparts is needed to ensure careful balance in managing both the psychiatric and motor symptoms in such patients. 